\title{
A susceptibility gene for type 2 diabetes confers substantial risk for diabetes complicating cystic fibrosis
}

\author{
S. M. Blackman • S. Hsu • S. E. Ritter • \\ K. M. Naughton • F. A. Wright • M. L. Drumm • \\ M. R. Knowles • G. R. Cutting
}

Received: 2 March 2009 / Accepted: 2 June 2009 /Published online: 8 July 2009

(C) Springer-Verlag 2009

\begin{abstract}
Aims/hypothesis Insulin-requiring diabetes affects 25-50\% of young adults with cystic fibrosis (CF). Although the cause of diabetes in $\mathrm{CF}$ is unknown, recent heritability studies in $\mathrm{CF}$ twins and siblings indicate that genetic modifiers play a substantial role. We sought to assess whether genes conferring risk for diabetes in the general population may play a risk modifying role in $\mathrm{CF}$.

Methods We tested whether a family history of type 2 diabetes affected diabetes risk in CF patients in 539 families
\end{abstract}

Electronic supplementary material The online version of this article (doi:10.1007/s00125-009-1436-2) contains supplementary material, which is available to authorized users.

S. M. Blackman · S. Hsu

Division of Pediatric Endocrinology,

Johns Hopkins University School of Medicine,

Baltimore, MD, USA

S. M. Blackman · S. E. Ritter · K. M. Naughton •

G. R. Cutting $(\bowtie)$

McKusick-Nathans Institute of Genetic Medicine,

Johns Hopkins University School of Medicine,

733 N. Broadway, Broadway Research Building 559,

Baltimore, MD 21205, USA

e-mail: gcutting@jhmi.edu

\section{F. A. Wright}

Department of Biostatistics, School of Public Health,

University of North Carolina at Chapel Hill,

Chapel Hill, NC, USA

\section{L. Drumm}

Departments of Pediatrics and Genetics,

Case Western Reserve University,

Cleveland, $\mathrm{OH}$, USA

\section{R. Knowles}

Cystic Fibrosis-Pulmonary Research and Treatment Center,

School of Medicine, University of North Carolina at Chapel Hill,

Chapel Hill, NC, USA in the CF Twin and Sibling family-based study. A type 2 diabetes susceptibility gene (transcription factor 7-like 2, or TCF7L2) was evaluated for association with diabetes in CF using 998 patients from the family-based study and 802 unrelated CF patients in an independent case-control study. Results Family history of type 2 diabetes increased the risk of diabetes in CF (OR 3.1; $p=0.0009$ ). A variant in TCF 7L2 associated with type 2 diabetes (the $\mathrm{T}$ allele at rs7903146) was associated with diabetes in CF in the family study $(p=0.004)$ and in the case-control study $(p=0.02$; combined $p=0.0002$ ). In the family-based study, variation in TCF $7 L 2$ increased the risk of diabetes about three-fold (HR 1.75 per allele, 95\% CI 1.3-2.4; $p=0.0006$ ), and decreased the mean age at diabetes diagnosis by 7 years. In $\mathrm{CF}$ patients not treated with systemic glucocorticoids, the effect of $T C F 7 L 2$ was even greater (HR 2.9 per allele, 95\% CI 1.7-4.9, $p=0.00011$ ).

Conclusions/interpretation A genetic variant conferring risk for type 2 diabetes in the general population is a modifier of risk for diabetes in $\mathrm{CF}$.

Keywords Association - CFRD - Corticosteroid . Cystic fibrosis · Diabetes · Genetics · Glucocorticoid . Modifier gene $\cdot$ TCF7L2
Abbreviations
CF Cystic fibrosis
MZ Monozygous
PI Pancreatic insufficiency
SNP Single-nucleotide polymorphism

\section{Introduction}

Diabetes is an important complication of cystic fibrosis (CF), a multisystem, genetic disease caused by mutations in 
the cystic fibrosis transmembrane conductance regulator gene (CFTR) [1]. Diabetes risk increases with age, affecting about $25 \%$ of adolescents and $40-50 \%$ of adults with CF $[2,3]$. As CF patients are now living longer, diabetes has become the most common systemic complication of $\mathrm{CF}$ after lung disease. Diabetes is associated with a significantly worse CF prognosis [4], although treatment of diabetes improves nutritional status and pulmonary function [5]. Among CF patients, the prevalence of diabetes is approximately tenfold greater (at about one-third the age) than is seen for type 2 diabetes in the general population [6].

About $90 \%$ of patients with CF have exocrine pancreatic insufficiency (PI), which is determined largely by CFTR mutation [7], and PI is a requirement for developing diabetes in CF [2, 3]. However, CFTR mutations do not otherwise explain diabetes risk; rather, we recently demonstrated that variation in other genes accounts for most of the risk for diabetes in $\mathrm{CF}$ [8]. Although type 1 and type 2 diabetes also have a strong genetic basis, it is unknown whether susceptibility genes for type 1 or type 2 diabetes might also play a role in CF. On one hand, there appears to be less similarity with type 1 diabetes, considering that diabetes incidence increases steadily with age rather than peaking in childhood, and that islet autoimmunity characteristic of type $1 \mathrm{~A}$ diabetes plays little or no role in $\mathrm{CF}$ according to most (e.g. Minicucci et al. [9], Lanng et al. [10]) but not all (e.g. Nousia-Arvanitakis et al. [11]) reports. On the other hand, type 2 diabetes, like diabetes in $\mathrm{CF}$, shows a monotonic increase in prevalence with age [6], a progressive defect in insulin secretion [12, 13], and an accumulation of amyloid polypeptide in pancreatic islets $[14,15]$. These similarities suggest that the disease mechanisms of diabetes in CF could be shared with type 2 diabetes seen in the general population.

In this study, we evaluated the relatives of CF patients to determine if diabetes in CF and type 2 diabetes cluster in the same families. We tested whether the rs7903146 variant in TCF7L2, the type 2 diabetes susceptibility gene whose common variant confers the greatest risk [16, 17], modifies risk of diabetes in $\mathrm{CF}$ and whether treatment with systemic glucocorticoids, an important non-genetic risk factor for diabetes in $\mathrm{CF}$, affected the risk conferred by TCF7L2.

\section{Methods}

Study participants Clinical data and DNA samples were collected by the Cystic Fibrosis Twin and Sibling familybased study [18, 19], with additional clinical data provided by the US CF Foundation Patient Registry (Bethesda, MD, USA). Enrolment was based on conclusive diagnosis of CF [20] and having an affected twin and/or sibling. As diabetes occurs almost exclusively in CF patients who are pancreatic insufficient [3], we selected $998 \mathrm{CF}$ patients with pancreatic exocrine insufficiency or a CFTR genotype predictive for severe pancreatic exocrine insufficiency (as described in [18]) from 1,154 patients $(86 \%)$ in the CF Twin and Sibling study for analysis (Table 1). Monozygous (MZ) twins were excluded to avoid oversampling of $\mathrm{MZ}$ transmission patterns. Each CF patient was classified using longitudinal clinical and laboratory data (described in [8]) as: euglycaemic (at least two diabetes screens done, all within normal limits); hyperglycaemic (milder glucose abnormalities such as impaired glucose tolerance); possible diabetes (e.g. intermittent or resolved diabetes); or diabetes (chronic, insulin-treated diabetes for $\geq 1$ year). Fifty-two were excluded (49 for insufficient information, three for antibody-positive type 1 diabetes).

Family history obtained by questionnaire [21] (available upon request) was stratified by degree of relatedness (e.g. first degree, such as a parent or sibling, vs second degree, such as a grandparent, aunt, or uncle) and was categorised as: positive (at least one first-degree or two second-degree relatives on the same side of the family giving a 'yes' response for 'Diabetes-Adult onset', recognising that about $90 \%$ of diabetic adults have type 2 diabetes [22]); possible (any other combination of second-degree relatives); or negative (no first- or second-degree relatives). A negative family history required completion of both nuclear and extended pedigree and no reported first- or second-degree relatives with adult-onset diabetes. The matched euglycaemic group of pancreatic insufficient euglycaemic patients was selected to match the diabetes patients by age and sex as described previously [8]. Other phenotypes were obtained from medical records, by questionnaire, and from the CF Foundation Patient Registry. Systemic glucocorticoid treatment was defined as oral or intravenous glucocorticoids (i.e. not intranasal or inhaled). Definitions of meconium ileus [18], lung function [19] and nutritional [23] phenotypes are described elsewhere.

The case-control study of unrelated CF patients used for replication is described elsewhere [24]. Briefly, the Genetic Modifiers Study (University of North Carolina/Case Western University) included $167 \mathrm{CF}$ patients with diabetes and $635 \mathrm{CF}$ patients without diabetes, all homozygous for the $\triangle F 508$ mutation in CFTR and affected with pancreatic exocrine insufficiency. Diabetes was defined as a diagnosis by a physician plus insulin use. This $\mathrm{CF}$ clinic sample was originally recruited based on age and lung function criteria: severe lung disease (260 patients of mean age 15.8 years; 38 diabetic); mild lung disease in an older age group (246 patients of mean age 37.7 years; 88 diabetic); and mild lung disease in a younger age group (296 patients of mean age 20.6 years; 41 diabetic) [24]. These studies were approved by the Institutional Review Boards of all participating 
Table 1 Comparison of clinical features of pancreatic insufficient CF patients (excluding MZ twins) in the twin/sibling study group with euglycaemia, hyperglycaemia or diabetes

\begin{tabular}{|c|c|c|c|}
\hline Characteristic & Euglycaemia & Hyperglycaemia or possible diabetes & Diabetes \\
\hline Number of participants & 509 & 387 & 102 \\
\hline Mean age (years) & $12.5^{\mathrm{h}}$ & $15.9^{\mathrm{h}}$ & 23.6 \\
\hline White & $476 / 509(94 \%)$ & $353 / 387(91 \%)$ & $96 / 102(94 \%)$ \\
\hline Female & $237 / 509(47 \%)$ & $175 / 387(45 \%)$ & $55 / 102(54 \%)$ \\
\hline \multicolumn{4}{|l|}{ CFTR genotype } \\
\hline$\Delta \mathrm{F} 508 \times 2^{\mathrm{a}}$ & $273 / 507(54 \%)$ & $218 / 386(56 \%)$ & $59 / 100(59 \%)$ \\
\hline Severe $C F T R \times 2^{\mathrm{b}}$ & $443 / 489(91 \%)$ & $356 / 369(95 \%)$ & $88 / 93(95 \%)$ \\
\hline \multicolumn{4}{|l|}{ Lung function percentile ${ }^{c}$} \\
\hline Cross-sectional & $0.73(n=391)^{\mathrm{h}}$ & $0.67(n=357)^{\mathrm{h}}$ & $0.55(n=98)$ \\
\hline Longitudinal & $0.63(n=253)^{\mathrm{h}}$ & $0.58(n=299)^{\mathrm{f}}$ & $0.51(n=94)$ \\
\hline \multicolumn{4}{|l|}{ Nutritional status ${ }^{\mathrm{d}}$} \\
\hline Cross-sectional & $-0.08(n=500)^{\mathrm{h}}$ & $-0.49(n=387)^{\mathrm{e}}$ & $-0.76(n=100)$ \\
\hline Longitudinal & $-0.04(n=492)^{\mathrm{h}}$ & $-0.29(n=382)$ & $-0.47(n=94)$ \\
\hline Steroid treatment ( $\geq 30$ days/year) & $90 / 509(18 \%)^{\mathrm{h}}$ & $78 / 387(20 \%)^{\mathrm{h}}$ & $46 / 102(45 \%)$ \\
\hline Steroid treatment (days/year) & $19(n=304)^{\mathrm{h}}$ & $31(n=268)^{\mathrm{h}}$ & $80(n=59)$ \\
\hline Positive test for Pseudomonas aeruginosa & $344 / 462(74 \%)^{\mathrm{h}}$ & $310 / 366(85 \%)^{\mathrm{g}}$ & $90 / 92(98 \%)$ \\
\hline Meconium ileus & $85 / 444(19 \%)^{\mathrm{e}}$ & $76 / 342(22 \%)^{\mathrm{f}}$ & $8 / 86(9 \%)$ \\
\hline Lung transplantation & $1 / 451(0.2 \%)^{\mathrm{h}}$ & $4 / 361(1 \%)^{\mathrm{g}}$ & $9 / 89(10 \%)$ \\
\hline
\end{tabular}

${ }^{\mathrm{a}}$ Homozygous for $\triangle \mathrm{F} 508$

${ }^{\mathrm{b}}$ Both CFTR alleles with severe (e.g. frameshift or nonsense) mutations (list available upon request)

${ }^{c}$ Cross-sectional and longitudinal measures of lung function were based on forced expiratory volume in $1 \mathrm{~s}$ as defined previously [19]

${ }^{\mathrm{d}}$ Nutritional status was assayed by body mass index standard deviation score (BMI SDS), with longitudinal averages as defined previously [23]. Cross-sectional BMI SDS was calculated by averaging over the year prior to enrolment

${ }^{\mathrm{e}} p<0.05,{ }^{\mathrm{f}} p<0.01,{ }^{\mathrm{g}} p<0.001,{ }^{\mathrm{h}} p<0.0001$ vs diabetes

institutions, and informed consent was obtained from all individuals before enrolment.

DNA isolation and genotyping DNA isolation and identification of CFTR mutations have been previously described [18]. TaqMan Assays-on-Demand (Applied Biosystems, Foster City, CA, USA) were used to genotype TCF7L2 single nucleotide polymorphism rs7903146 as described elsewhere [23]. Genotype repeatability was $99.7 \%$ in 1,133 duplicated samples. One Mendelian error was identified in 678 families in the family-based study and was corrected by DNA sequencing. DNA from those with absent or erroneous genotypes was sequenced using the BigDye Terminator v3.1 Cycle Sequencing Kit (Applied Biosystems). Genotypes were verified by sequencing in $2 \%$ of the samples; no discrepancies were detected.

Statistical analysis Genetic association was deemed present when the frequency of allele inheritance differed significantly from random Mendelian transmission of alleles. Transmission distortion was quantified using the FBAT statistic as implemented in the software packages FBAT 1.7.3 [25] and PBAT 3.5 [26] with an additive genetic model and an optimal phenotypic offset [27]. Essentially identical results were obtained using an offset equal to the prevalence rate of diabetes in the analysed sample and offsets of 0 (considering only affected individuals) or 1 (considering only unaffected individuals). Age of onset of diabetes was analysed as a censored trait using PBAT. Genetic association was also assessed after disregarding family structure by comparison of genotype and allele frequencies with diabetes status (case-control association analysis) and with diabetes-free survival rates (association with cumulative incidence of disease). The same results were obtained when analysis was restricted to individuals who were not informative in the family-based analysis (not shown). Populationattributable risk was calculated from hazard ratios (e.g. Altshuler et al. [28]).

Statistical calculations were performed using Intercooled Stata 10 (StataCorp, College Station, TX, USA). Where there were small cell sizes, the Fisher exact test was used. Student's $t$ test was used to compare normally distributed continuous data. The logrank test was used to compare cumulative incidence rates. Significance levels from analysis of the primary (family-based) and replication 
(case-control) studies were combined calculating a average of the $Z$ statistics weighted by the square root of the number of individuals in each study (e.g. Gaulton et al. [29]).

\section{Results}

Diabetes in a first-degree relative correlated with diabetes in the CF patient (OR 2.8, 95\% CI 1.2-6.1, $p=0.01$; Table 2). Diabetes in at least two second-degree relatives on the same side of the family trended toward correlation (OR 2.2, 95\% CI $0.93-5.1, p=0.07)$. CF patients meeting either of these two conditions had an increased risk for diabetes in CF (OR $2.8,95 \%$ CI $1.5-5.3, p=0.001)$. Family history was known for about half the study group and did not depend on the CF patient's diabetes status. Because our CF patients with diabetes were, on average, older than other CF patients in this study, we examined whether the greater risk for familial diabetes might be accounted for by the older age of family members. This was not the case, as the correlation between a positive family history of diabetes (especially in a firstdegree relative) and diabetes in $\mathrm{CF}$ remained substantial when diabetic CF patients were compared with euglycaemic CF patients matched for age, sex and pancreatic status (matched euglycaemic; Table 2).

Genotypes of the TCF7L2 single-nucleotide polymorphism (SNP) (rs7903146) were obtained for 943 of the 998 available CF patients, providing 533 parent-parent-child trios for analysis. Parental genotypes were in HardyWeinberg equilibrium $(p=0.7)$, and their minor allele frequency $(28 \%)$ was in the range reported for white populations [30]. In families, the $\mathrm{T}$ allele of the TCF7L2 SNP was transmitted to CF patients with diabetes significantly more often than would be expected by chance, demonstrating association with increased risk of diabetes (200 informative families, $p=0.005$; FBAT). Distorted transmission was also seen in separate analyses of the diabetic ( $\mathrm{T}$ allele overtransmitted, 54 informative families, $p=0.04$ ) and euglycaemic (C allele overtransmitted, 146 informative families, $p=0.01$; FBAT) subgroups, demonstrating that variation in the TCF7L2 SNP operates at both phenotypic extremes. Finally, diabetic CF patients displayed substantial differences from euglycaemic $\mathrm{CF}$ patients in the frequency of the high-risk TCF7L2 SNP. As shown in Electronic supplementary material (ESM) Table 1, differences were seen in allele frequency ( $p=0.007)$ and genotype frequency $(p=0.02$ for the additive model, $p=0.009$ for the dominant model) that yielded a 1.7 fold increase in disease odds per allele (95\% CI 1.5-2.4, $p=0.007$ ).

Variation at TCF7L2 also affected the age at which diabetes was diagnosed. Treating the diabetes diagnosis as a time-dependent event (by censoring at the present age), we noted that CF patients in the family-based study who had inherited the $\mathrm{T}$ allele of TCF7L2 developed diabetes at a younger age than those who inherited the $\mathrm{C}$ allele (211 informative families, logrank $p=0.01$; Wilcoxon $p=0.002$, PBAT). The magnitude of this effect is estimated by comparing diabetes incidence with TCF7L2 genotype for all $\mathrm{CF}$ patients in the family study, disregarding family structure (Fig. 1a). Each high-risk $\mathrm{T}$ allele of TCF7L2 conferred a significant increase in the cumulative incidence of diabetes (logrank $p=0.001$; HR 1.75, 95\% CI 1.3-2.4; $p=0.0006$ ). While it is possible that specific mutations of the CFTR gene could affect diabetes risk, similar results were obtained when analysis was restricted to the 518 individuals homozygous for $\triangle \mathrm{F} 508$, the most common CFTR genotype (HR 1.5 per allele, $95 \%$ CI $1.0-2.3, p=0.049)$ or the other 439 individuals (not homozygous for $\triangle \mathrm{F} 508$ ) with two mutations causing severe CFTR dysfunction (e.g. frameshift or nonsense mutations; HR 2.17, 95\% CI 1.3-3.6, $p=0.003$; difference in HR not significant, $p=0.2$ ).

Association of the TCF7L2 SNP with diabetes in CF was also seen in an independent collection of unrelated $\mathrm{CF}$ patients recruited originally based on age and lung disease by the UNC/CWRU Genetic Modifiers Study [24]. In that

Table 2 Influence of a family history of type 2 diabetes on diabetes prevalence in pancreatic insufficient CF patients

\begin{tabular}{|c|c|c|c|c|c|}
\hline Family history & Diabetic & Euglycaemic & OR $(95 \% \mathrm{CI})^{\mathrm{a}}$ & $\begin{array}{l}\text { Matched } \\
\text { euglycaemic }^{\text {b }}\end{array}$ & OR $(95 \% \mathrm{CI})^{\mathrm{c}}$ \\
\hline Both first- and second-degree family history known & $65 / 128(51 \%)$ & $274 / 500(55 \%)$ & $0.85(0.6-1.3)$ & $68 / 128(53 \%)$ & $0.91(0.5-1.4)$ \\
\hline First-degree relative with diabetes & $11 / 78(14 \%)$ & $18 / 320(6 \%)$ & $2.8(1.2-6.1)^{\mathrm{d}}$ & $2 / 83(2 \%)$ & $6.6(1.4-31)^{\mathrm{d}}$ \\
\hline $\begin{array}{l}\text { Two or more second-degree relatives on the same side } \\
\text { with diabetes }\end{array}$ & $9 / 65(14 \%)$ & 19/276 (7\%) & $2.2(0.93-5.1)$ & $5 / 68(7 \%)$ & $2.0(0.6-6.4)$ \\
\hline $\begin{array}{l}\text { One or more first- or two or more second-degree } \\
\text { relatives on the same side with diabetes }\end{array}$ & $19 / 78(24 \%)$ & $33 / 321(10 \%)$ & $2.8(1.5-5.3)^{\mathrm{f}}$ & $7 / 83(8 \%)$ & $3.5(1.4-9)^{\mathrm{e}}$ \\
\hline
\end{tabular}

${ }^{\text {a }}$ OR $(95 \% \mathrm{CI})$ comparing family history, diabetic group vs euglycaemic group

${ }^{\mathrm{b}}$ Diabetes patients were matched by age, sex and number of $\Delta \mathrm{F} 508$ alleles with euglycaemic patients

${ }^{\mathrm{c}} \mathrm{OR}(95 \% \mathrm{CI})$ comparing family history, diabetic group vs matched euglycaemic group

${ }^{\mathrm{d}} p<0.05,{ }^{\mathrm{e}} p<0.01,{ }^{\mathrm{f}} p=0.001$ vs diabetic group 


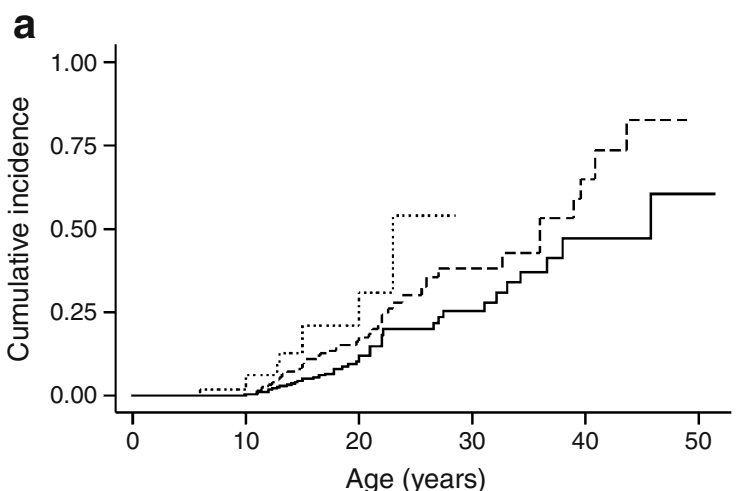

Number at risk:

$\begin{array}{ccccccc}\mathrm{C} / \mathrm{C} & 479 & 336 & 104 & 31 & 6 & 2 \\ \mathrm{C} / \mathrm{T} & 392 & 276 & 78 & 17 & 5 & 1 \\ \mathrm{~T} / \mathrm{T} & 72 & 43 & 8 & 0 & 0 & 0\end{array}$

Fig. 1 Cumulative incidence of diabetes grouped by TCF7L2 SNP genotype. Individuals in the primary (family-based) study (a) who were homozygous for the major allele (C/C genotype, solid line) developed diabetes at a significantly lower rate than patients heterozygous $(\mathrm{C} / \mathrm{T}$ genotype, dashed line) or homozygous for the minor allele ( $\mathrm{T} / \mathrm{T}$ genotype, dotted line). Results were similar when comparing all three genotypes separately as shown (additive genetic model; $p=0.001$, logrank) or when grouping genotypes according to dominant $(p=0.003)$ or recessive $(p=0.007)$ genetic models. Modelling of these data indicated that each $\mathrm{T}$ allele conferred about a $75 \%$ increase in risk of developing diabetes compared with PI CF patients of the same age without a T allele (HR 1.75, 95\% CI 1.3-2.4; $p=0.0006$ ). The age at which the cumulative incidence reached $25 \%$ varied by 7 years with the number of high-risk $\mathrm{T}$ alleles $(\mathrm{C} / \mathrm{C}$

study, those at greatest risk for diabetes (because of increased age) are a subgroup of 246 patients recruited for older age and milder lung disease (88 with diabetes, 158 without diabetes). In the older group, a significant increase in risk for those carrying a $\mathrm{T}$ allele was evident at both the allele (OR 1.6, 95\% CI 1.1-2.4, $p=0.02$ ) and genotype levels (OR 1.9, 95\% CI 1.1-3.6, $p=0.016$; dominant model) and by regression analysis (per-allele OR 1.92, 95\% CI $1.1-3.3, p=0.015$ ) as shown in ESM Table 1. Genotype frequencies in two subgroups of younger patients (with mild or severe lung disease) from the UNC/CWRU study that are predicted to be at lower risk for diabetes did not show association with diabetes. When age of onset of diabetes was considered, there was an increase in diabetes cumulative incidence with one or two TCF $2 L 2 \mathrm{~T}$ alleles in both the older age subgroup (logrank $p=0.0035$; HR 1.9, $95 \%$ CI 1.2-2.9, $p=0.004$, dominant model), and when all 802 unrelated patients were considered (logrank $p=0.02$; HR 1.4, 95\% CI 1.1-2.0, $p=0.02$, Fig. 1b). Thus, the T allele of the TCF7L2 SNP conferred increased risk and earlier age at diagnosis for diabetes in the family-based and case-control studies. Considered jointly, the two studies support association of the rs7903146 SNP of TCF7L2 with diabetes in CF $\left(p=2.0 \times 10^{-4}\right)$.

\section{b}

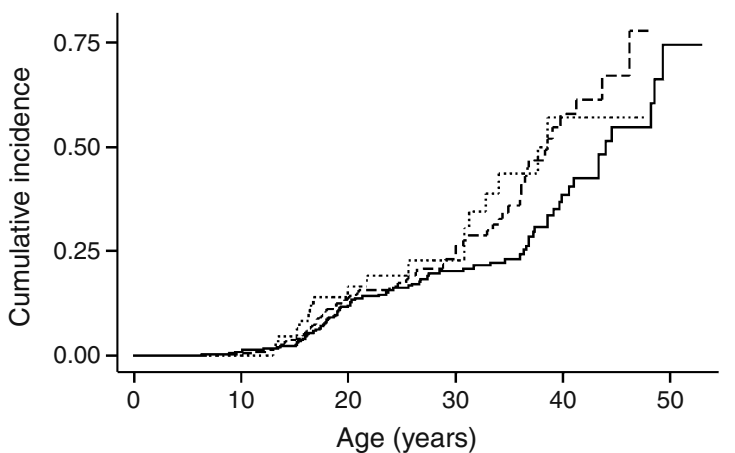

Number at risk:

$\begin{array}{ccccccc}\mathrm{C} / \mathrm{C} & 402 & 389 & 221 & 121 & 37 & 2 \\ \mathrm{C} / \mathrm{T} & 330 & 324 & 162 & 67 & 13 & 0 \\ \mathrm{~T} / \mathrm{T} & 70 & 70 & 34 & 20 & 5 & 0\end{array}$

genotype: 27.4 years; $\mathrm{C} / \mathrm{T}$ genotype: 22.6 years; $\mathrm{T} / \mathrm{T}$ genotype: 20.0 years). Individuals in the replication (case-control) study (b) who were homozygous for the major allele (C/C genotype, solid line) developed diabetes at a significantly lower rate than patients with one or two copies of the minor allele $(\mathrm{C} / \mathrm{T}$ genotype, dashed line; or $\mathrm{T} / \mathrm{T}$ genotype, dotted line). In this study (which had a targeted recruitment strategy based on age and lung function) the data were more consistent with a dominant genetic model, with incidence rates for all three genotypes considered separately being less distinct ( $p=0.06$, logrank), and rates for those with one or two copies of the $\mathrm{T}$ allele being significantly greater than for those with none $(p=0.02$, logrank). Modelling of these data showed association under dominant (HR 1.43, $95 \%$ CI $1.1-2.0 ; p=0.02$ ) or additive (HR 1.3, 95\% CI 1.04-1.61, $p=0.02$ ) genetic models

We tested whether an important non-genetic risk factor for diabetes in $\mathrm{CF}$, treatment with systemic glucocorticoids ([8, 31, 32] and Table 1), may interact with TCF7L2. Regression modelling in the family-based study suggested an interaction between steroid treatment and TCF7L2 genotype (ESM Table 2). That is, TCF7L2 did not significantly affect diabetes risk in the $405 \mathrm{CF}$ patients receiving glucocorticoid treatment in the last year (HR 1.4 per allele, 95\% CI 0.9-2.1, $p=0.14$; ESM Fig. 1) or in the $103 \mathrm{CF}$ patients treated with glucocorticoids for at least 30 days (HR 1.1 per allele, 95\% CI 0.6-2.0, $p=0.8$ ), whereas the risk ratio for TCF $T 22$ was greater in the other 485 patients who were not treated with glucocorticoids in the last year (HR 2.9 per allele, 95\% CI 1.7-4.9, $\mathrm{p}=1.1 \times 10^{-4}$; ESM Fig. 2). The TCF7L2 SNP remained an independent risk factor with essentially the same effect size after adjusting for cross-sectional or longitudinal measures of lung function, body mass index, and family history of type 2 diabetes (data not shown). Data were not available to perform a similar analysis in the replication (case-control) study. In summary, adjusting for steroid exposure, an important clinical risk factor for diabetes in $\mathrm{CF}$, reveals a subgroup in which the effect size of TCF7L2 association is substantially increased. 


\section{Discussion}

Diabetes in CF patients and type 2 diabetes in the general population share some pathophysiological features, and both have a strong genetic basis. This multicentre study demonstrates, with independent replication, that a susceptibility gene for type 2 diabetes (TCF7L2) plays a role in modifying the risk for diabetes in CF. Family history data suggest that other susceptibility genes for type 2 diabetes may also play a role. Finally, a non-genetic factor, treatment with systemic glucocorticoids, modifies the risk conferred by $T C F 7 L 2$. In the first analysis (family history), the increased diabetes risk to the CF patient depended on the number of and genetic distance to affected relatives, as is the case for type 2 diabetes [33], suggesting that familial sharing of genes is more important than sharing of environment. Two prior studies found no significant correlation between diabetes in $\mathrm{CF}$ and diabetes in family members without CF $[9,10]$. Neither study was powered to detect effects of the magnitude measured in the current study, although one found a trend toward correlation [9].

From the validated susceptibility genes for type 2 diabetes, we selected TCF7L2 for testing based on it having the greatest effect size and its validation in the largest number of patients [34]. Greater diabetes risk was associated with the same TCF7L2 allele in CF as in the general population [16], indicating that this genetic risk factor is shared by these two forms of diabetes. Decreased pancreatic beta cell function is a hallmark of diabetes in $\mathrm{CF}$ (e.g. Moran et al. [2], Bismuth et al. [13]), so the involvement of TCF7L2 in CF supports roles hypothesised for TCF7L2 in beta cell proliferation and/or insulin secretion (e.g. Liu et al. [35], Wegner et al. [36]). We hypothesise that variation in TCF7L2 affects beta cell function in CF leading to earlier development of diabetes, as is predicted to occur in type 2 diabetes [37]. However, the effect of TCF7L2 (relative risk of 1.42-1.51 per allele in the general population [30]) may be greater in some pancreatic insufficient $\mathrm{CF}$ patients, as suggested by the greater risk ratio seen in those patients not treated with systemic steroids (95\% CI 1.7-4.9). Using the hazard ratio in Fig. 1 to estimate effect size, variation at TCF7L2 is estimated to accounted for a substantial fraction of the diabetes cases in the current $\mathrm{CF}$ study (population-attributable risk 32\%, 95\% CI 14-49\%). The cumulative incidence analyses may underestimate the magnitude of TCF7L2 effect, because hyperglycaemic and possible diabetes patients were grouped with euglycaemic patients as a consequence of lack of age-of-onset data for these intermediate phenotypes. To illustrate the effect of combining this TCF7L2 variant and CFTR dysfunction, we note that a 14-year-old CF patient with two high-risk TCF7L2 alleles has the same predicted risk for diabetes as a 60 -year- old individual in the general population with no TCF7L2 risk alleles [6], a difference of 46 years attributable to variation in both CFTR and TCF7L2.

In the general population, the risk of type 2 diabetes conferred by TCF $7 L 2$ appears to be reduced by interaction with environmental factors such as lifestyle intervention [37]. In CF, the current study found evidence that treatment with systemic steroids influences the effect of TCF7L2 on risk for diabetes by 1.7-fold. Accounting for sources of environmental variation such as steroid treatment made the remaining sources of variation in $\mathrm{CF}$ patients (such as from genetic modifiers) more prominent. While there has been debate as to the utility of stratifying risk for type 2 diabetes by typing individuals for susceptibility genes (e.g. Meigs et al. [38], Lyssenko et al. [39]), in the case of CF patients not treated with steroids, a substantially earlier predicted onset for diabetes is likely to be clinically relevant, and could be used to individualise the timing of diabetes screening and initiation of treatment.

The strengths of the current study include the use of family-based association testing which is insensitive to artefacts generated by population stratification [25], and the depth and breadth of longitudinal clinical data (collected as part of CF care and largely unrelated to whether patients were diagnosed with diabetes). A limitation is that not all patients received regular oral glucose tolerance testing. We sought to minimise the effect of this limitation by excluding individuals with insufficient data to classify their phenotype, and by using additional information such as postprandial glucose or $\mathrm{HbA}_{1 \mathrm{c}}$ data. Because the highly detailed phenotype information was not available for the replication (case-control) study, some individuals may have been misclassified as not having diabetes in that study, reducing the apparent effect of TCF7L2 in that sample. This consideration and the targeted recruitment strategy used in the latter study limit the degree to which quantitative comparisons of TCF7L2 effects may be made between the primary and replication studies. Finally, it should be recognised that this study's findings are limited to the $\sim 90 \%$ of CF patients who have severe exocrine pancreatic insufficiency, as the $\sim 10 \%$ of CF patients with significant pancreatic exocrine function are not at high risk for developing diabetes.

This study demonstrates diabetes in CF and type 2 diabetes have at least one pathological mechanism in common. The higher frequency and earlier development of diabetes in CF patients present an opportunity to evaluate and optimise gene-based tests or therapies that may have implications for individuals with type 2 diabetes. On the other hand, the clinical differences exemplified by the underweight young adult with $\mathrm{CF}$ and diabetes and the older, often overweight, adult with type 2 diabetes may be exploited to investigate how variation in genes such as 
TCF7L2 lead to diabetes in different nutritional and inflammatory contexts. Thus, characterisation of genetic modifiers of monogenic disorders like CF may help inform the mechanisms by which common polymorphisms cause common diseases such as type 2 diabetes.

Acknowledgements The authors gratefully acknowledge the participation of the many CF patients, families, research coordinators and clinicians in the CF Twin and Sibling Study, the Genetic Modifiers of CF Study, and the Canadian Consortium for CF Genetic Studies. Portions of this work have been published previously in abstract form at the 2007 North American Cystic Fibrosis Conference and the 2007 meeting of the American Society of Human Genetics. The JHU CF Twin and Sibling Study is supported by NIH grant DK076446 (S. M. Blackman), DK044003 and HL068927 (G. R. Cutting) and CF Foundation funding CUTTIN06P0 (G. R. Cutting). The UNC/CWRU Genetic Modifiers Study is supported by the CF Foundation (KNOWLE00A0) and NIH grants HL068890, DK066368 (M. R. Knowles) and RR000046. The funding sources had no role in the study design, collection, analysis, and interpretation of data, writing of the report, or the decision to submit for publication. The authors thank L. L. Vanscoy, J. M. Collaco, L. B. Henderson and D. Green for assistance in defining important clinical covariates in the JHU study, P. Sosnay and K. McDougal for helpful discussions, P. Cornwall for administrative help, K. Yao for aid in SNP testing, and D. J. Cutler for guidance in data analysis. The authors also thank R. Pace for coordination of the UNC/CWRU molecular biology of the study; K. Mohlke for advice about SNP selection; J. Luo, Director of the Human Molecular Genotyping Core, for SNP testing; S. Wood and L. Charnin for coordination of the clinical phenotyping; H. Calloway for assistance in data analysis and illustrations; D. Fargo and A. Xu for Bioinformatics support; and the Gene Modifier Study Group. The authors also thank P. Durie, R. Dorfman, J. Zielenski, and M. Corey (Hospital for Sick Children, Toronto, Canada) for proofreading the manuscript. The authors declare that there is no duality of interest associated with this manuscript.

\section{References}

1. Welsh MJ, Ramsey BW, Accurso FJ, Cutting GR (2001) Cystic fibrosis. In: Scriver CR, Beaudet AL, Valle D, Sly WS (eds) The metabolic and molecular bases of inherited disease, 8th edn. McGraw-Hill, New York, pp 5121-5188

2. Moran A, Hardin D, Rodman D et al (1999) Diagnosis, screening and management of cystic fibrosis related diabetes mellitus: a consensus conference report. Diabetes Res Clin Pract 45:61-73

3. Mackie AD, Thornton SJ, Edenborough FP (2003) Cystic fibrosis-related diabetes. Diabet Med 20:425-436

4. Milla CE, Warwick WJ, Moran A (2000) Trends in pulmonary function in patients with cystic fibrosis correlate with the degree of glucose intolerance at baseline. Am J Respir Crit Care Med 162:891-895

5. Mohan K, Israel KL, Miller H, Grainger R, Ledson MJ, Walshaw MJ (2008) Long-term effect of insulin treatment in cystic fibrosisrelated diabetes. Respiration 76:181-186

6. Cowie CC, Rust KF, Byrd-Holt DD et al (2006) Prevalence of diabetes and impaired fasting glucose in adults in the U.S. population: National Health and Nutrition Examination Survey 1999-2002. Diabetes Care 29:1263-1268

7. Ahmed N, Corey M, Forstner G et al (2003) Molecular consequences of cystic fibrosis transmembrane regulator (CFTR) gene mutations in the exocrine pancreas. Gut 52:1159-1164
8. Blackman SM, Hsu S, Vanscoy LL et al (2009) Genetic modifiers play a substantial role in diabetes complicating cystic fibrosis. J Clin Endocrinol Metab 94:1302-1309

9. Minicucci L, Cotellessa M, Pittaluga L et al (2005) Beta-cell autoantibodies and diabetes mellitus family history in cystic fibrosis. J Pediatr Endocrinol Metab 18:755-760

10. Lanng S, Thorsteinsson B, Pociot F et al (1993) Diabetes mellitus in cystic fibrosis: genetic and immunological markers. Acta Paediatr 82:150-154

11. Nousia-Arvanitakis S, Galli-Tsinopoulou A, Dracoulacos D, Karamouzis M, Demitriadou A (2000) Islet autoantibodies and insulin dependent diabetes mellitus in cystic fibrosis. J Pediatr Endocrinol Metab 13:319-324

12. Kahn SE (2001) Clinical review 135: The importance of beta-cell failure in the development and progression of type 2 diabetes. J Clin Endocrinol Metab 86:4047-4058

13. Bismuth E, Laborde K, Taupin P et al (2008) Glucose tolerance and insulin secretion, morbidity, and death in patients with cystic fibrosis. J Pediatr 152(540-5):545

14. Hull RL, Westermark GT, Westermark P, Kahn SE (2004) Islet amyloid: a critical entity in the pathogenesis of type 2 diabetes. J Clin Endocrinol Metab 89:3629-3643

15. Couce M, O'Brien TD, Moran A, Roche PC, Butler PC (1996) Diabetes mellitus in cystic fibrosis is characterized by islet amyloidosis. J Clin Endocrinol Metab 81:1267-1272

16. Grant SF, Thorleifsson G, Reynisdottir I et al (2006) Variant of transcription factor 7-like 2 (TCF7L2) gene confers risk of type 2 diabetes. Nat Genet 38:320-323

17. Weedon MN (2007) The importance of TCF7L2. Diabet Med 24:1062-1066

18. Blackman SM, Deering-Brose R, McWilliams R et al (2006) Relative contribution of genetic and nongenetic modifiers to intestinal obstruction in cystic fibrosis. Gastroenterology 131:1030-1039

19. Vanscoy LL, Blackman SM, Collaco JM et al (2007) Heritability of lung disease severity in cystic fibrosis. Am J Respir Crit Care Med 175:1036-1043

20. Rosenstein BJ, Cutting GR (1998) The diagnosis of cystic fibrosis: a consensus statement. J Pediatr 132:589-595

21. Kahn LB, Marshall JA, Baxter J, Shetterly SM, Hamman RF (1990) Accuracy of reported family history of diabetes mellitus. Results from San Luis Valley Diabetes Study. Diabetes Care 13:796-798

22. Zimmet P, Alberti KG, Shaw J (2001) Global and societal implications of the diabetes epidemic. Nature 414:782-787

23. Collaco JM, Vanscoy L, Bremer L et al (2008) Interactions between secondhand smoke and genes that affect cystic fibrosis lung disease. JAMA 299:417-424

24. Drumm ML, Konstan MW, Schluchter MD et al (2005) Genetic modifiers of lung disease in cystic fibrosis. $\mathrm{N}$ Engl $\mathrm{J}$ Med 353:1443-1453

25. Laird NM, Horvath S, Xu X (2000) Implementing a unified approach to family-based tests of association. Genet Epidemiol 19 (Suppl 1):S36-S42

26. Steen KV, Lange C (2005) PBAT: a comprehensive software package for genome-wide association analysis of complex familybased studies. Hum Genome 2:67-69

27. Lunetta KL, Faraone SV, Biederman J, Laird NM (2000) Familybased tests of association and linkage that use unaffected sibs, covariates, and interactions. Am J Hum Genet 66:605-614

28. Altshuler D, Hirschhorn JN, Klannemark M et al (2000) The common PPARgamma Pro12Ala polymorphism is associated with decreased risk of type 2 diabetes. Nat Genet 26:76-80

29. Gaulton KJ, Willer CJ, Li Y et al (2008) Comprehensive association study of type 2 diabetes and related quantitative traits with 222 candidate genes. Diabetes 57:3136-3144 
30. Cauchi S, El Achhab Y, Choquet $\mathrm{H}$ et al (2007) TCF7L2 is reproducibly associated with type 2 diabetes in various ethnic groups: a global meta-analysis. J Mol Med 85:777-782

31. Marshall BC, Butler SM, Stoddard M, Moran AM, Liou TG, Morgan WJ (2005) Epidemiology of cystic fibrosis-related diabetes. J Pediatr 146:681-687

32. Adler AI, Gunn E, Haworth CS, Bilton D (2007) Characteristics of adults with and without cystic fibrosis-related diabetes. Diabet Med 24:1143-1148

33. Weijnen CF, Rich SS, Meigs JB, Krolewski AS, Warram JH (2002) Risk of diabetes in siblings of index cases with Type 2 diabetes: implications for genetic studies. Diabet Med 19:41-50

34. Helgason A, Palsson S, Thorleifsson G et al (2007) Refining the impact of TCF7L2 gene variants on type 2 diabetes and adaptive evolution. Nat Genet 39:218-225
35. Liu Z, Habener JF (2008) Glucagon-like peptide-1 activation of TCF7L2-dependent Wnt signaling enhances pancreatic beta-cell proliferation. J Biol Chem 283(13):8723-8735

36. Wegner L, Hussain MS, Pilgaard K et al (2008) Impact of TCF7L2 rs7903146 on insulin secretion and action in young and elderly Danish twins. J Clin Endocrinol Metab 93:4013-4019

37. Florez JC, Jablonski KA, Bayley N et al (2006) TCF7L2 polymorphisms and progression to diabetes in the Diabetes Prevention Program. N Engl J Med 355:241-250

38. Meigs JB, Shrader P, Sullivan LM et al (2008) Genotype score in addition to common risk factors for prediction of type 2 diabetes. N Engl J Med 359:2208-2219

39. Lyssenko V, Jonsson A, Almgren P et al (2008) Clinical risk factors, DNA variants, and the development of type 2 diabetes. N Engl J Med 359:2220-2232 